\title{
CONTAINMENTS OF SYMBOLIC POWERS OF IDEALS OF GENERIC POINTS IN $\mathbb{P}^{3}$
}

\author{
MARCIN DUMNICKI
}

(Communicated by Lev Borisov)

\begin{abstract}
We show that the Conjecture of Harbourne and Huneke, stating that $I^{(N r-(N-1))} \subset M^{(r-1)(N-1)} I^{r}$ for ideals of points in $\mathbb{P}^{N}$, holds for generic (simple) points for $N=3$. As a result, for such ideals we prove the following bounds, which can be recognized as generalizations of Chudnovsky bounds: $\alpha\left(I^{(3 m-k)}\right) \geq m \alpha(I)+2 m-k$, for any $m \geq 1$ and $k=0,1,2$. Moreover, we obtain lower bounds for the Waldschmidt constant for such ideals.
\end{abstract}

\section{INTRODUCTION}

Let $\mathbb{K}$ be a field of chracteristic zero, and let $\mathbb{K}\left[\mathbb{P}^{N}\right]=\mathbb{K}\left[x_{0}, \ldots, x_{N}\right]$ denote the ring of coordinates of the projective space with standard grading. Let $I \subset \mathbb{K}\left[\mathbb{P}^{N}\right]$ be a homogeneous ideal. By the $m$-th symbolic power we mean

$$
I^{(m)}=\mathbb{K}\left[\mathbb{P}^{N}\right] \cap\left(\bigcap_{\mathfrak{p} \in \operatorname{Ass}(I)} I^{m} \mathbb{K}\left[\mathbb{P}^{N}\right]_{\mathfrak{p}}\right),
$$

where the intersection is taken in the ring of fractions of $\mathbb{K}\left[\mathbb{P}^{N}\right]$. By a fat points ideal we mean the ideal

$$
I=\bigcap_{j=1}^{n} \mathfrak{m}_{p_{j}}^{m_{j}}
$$

where $\mathfrak{m}_{p}$ denotes the ideal of forms vanishing at a point $p \in \mathbb{P}^{N}$ and $m_{j} \geq 1$ are integers. Observe that for a fat points ideal $I$ as above,

$$
I^{(m)}=\bigcap_{j=1}^{n} \mathfrak{m}_{p_{j}}^{m_{j} m} .
$$

Let $M=\left(x_{0}, \ldots, x_{N}\right) \subset \mathbb{K}\left[\mathbb{P}^{N}\right]$ be the maximal homogeneous ideal.

A sequence $\bar{m}=\left(m_{1}, \ldots, m_{n}\right)$ of $n$ integers will be called a sequence of multiplicities. Define the ideal of generic fat points in $\mathbb{K}\left[\mathbb{P}^{N}\right]$ to be

$$
I(\bar{m})=I\left(m_{1}, \ldots, m_{n}\right)=\bigcap_{j=1}^{n} \mathfrak{m}_{p_{j}}^{m_{j}}
$$

for generic points $p_{1}, \ldots, p_{n}$ in $\mathbb{P}^{N}$ (for $m_{j}<0$ we take $\mathfrak{m}_{p_{j}}^{m_{j}}=\mathbb{K}\left[\mathbb{P}^{N}\right]$ ). We will use the following notation:

$$
m^{\times s}=\underbrace{(m, \ldots, m)}_{s} \text {. }
$$

Received by the editors November 29, 2012 and, in revised form, May 17, 2013.

2010 Mathematics Subject Classification. Primary 14Q10, 13P10.

Key words and phrases. Symbolic powers, fat points. 
If all multiplicities are equal to one, we say that $I$ is an ideal of generic points or that we are interested in generic points.

In Ein-Laz-Smi 01 it is shown that $I^{(N r)} \subset I^{r}$ for any radical homogeneous ideal $I \subset \mathbb{K}\left[\mathbb{P}^{N}\right]$. This result has been improved in a sequence of papers by Boc-Har 10a, PSC 09, Har-Hun 11] and the author to the following:

Theorem 1. Let I be an ideal of a finite number of generic points in $\mathbb{P}^{N}$. Then:

- $I^{(3)} \subset I^{2}$ for $N=2($ Boc-Har 10a $)$,

- $I^{(2 r-1)} \subset I^{r}$ for $N=2([$ PSC 09] $)$,

- $I^{(2 r)} \subset M^{r} I^{r}$ for $N=2([$ Har-Hun 11] $)$,

- $I^{(3 r)} \subset M^{2 r} I^{r}$ for $N=3$ (Dum 11),

- $I^{(2 r-1)} \subset M^{r-1} I^{r}$ for $N=2([$ Har-Hun 11] $)$,

- $I^{(N r-(N-1))} \subset I^{r}$, where the number of points is sufficiently big (depending on $N)$ ([י PSC 09]).

It is also conjectured that

Conjecture 2. Let I be an ideal of points in $\mathbb{P}^{N}$. Then:

- $I^{(N r-(N-1))} \subset I^{r}$ (Conjecture 4.1 in Har-Hun 11),

- $I^{(N r-(N-1))} \subset M^{(r-1)(N-1)} I^{r}$ (Conjecture 4.5 in Har-Hun 11])

for all $r \geq 1$.

In the paper we show that Conjecture 2 holds for any number of generic points in $\mathbb{P}^{3}$.

Theorem 3. Let $I$ be an ideal of generic points in $\mathbb{P}^{3}$. Then $I^{(3 r-2)} \subset M^{2 r-2} I^{r}$ for any $r \geq 1$; thus also $I^{(3 r-2)} \subset I^{r}$ for any $r \geq 1$. Additionally, we have $I^{(3 r-1)} \subset M^{2 r-1} I^{r}$ for any $r \geq 1$, which, together with the main result in Dum 11. $\left(I^{(3 r)} \subset M^{2 r} I^{r}\right)$, completes the picture.

Proof. The proof of the theorem will be divided into six separated cases, depending on the number of points $n$ and $r$. Proposition [15] deals with $n \geq 512, r \geq 3$, Proposition 16 with $5 \leq n \leq 511, r \geq 3$, Proposition 17 with $n \geq 65, r=2$, Proposition 18 with $7 \leq n \leq 64, r=2$, Lemma 19 with $n=5,6, r=2$, and Proposition 22 with $n \leq 4$ and arbitrary $r$. The case $r=1$ is trivial.

For a homogeneous ideal $I \subset \mathbb{K}\left[\mathbb{P}^{N}\right]$ define the homogeneous part of degree $t$,

$$
I_{t}=\{f \in I: \operatorname{deg}(f)=t\},
$$

and the initial degree,

$$
\alpha(I)=\min \left\{t \geq 0: I_{t} \neq 0\right\} .
$$

Observe that $I_{t}$ is a $\mathbb{K}$-vector space.

Remark 4. Chudnovsky Chud 81] conjectured that for an ideal of points in $\mathbb{P}^{3}$ the following bound holds:

$$
\alpha\left(I^{(r)}\right) \geq r \frac{\alpha(I)+2}{3} .
$$

This bound follows from the containment $I^{(3 r)} \subset M^{2 r} I^{r}$ (see [Har-Hun 11]), which has been proved in Dum 11] for ideals of generic points. Moreover, this containment obviously gives

$$
\alpha\left(I^{(3 r)}\right) \geq r \alpha(I)+2 r .
$$


Observe that the containment $I^{(3 r-k)} \subset M^{2 r-k} I^{r}$ gives the following bound for $\alpha\left(I^{(3 r-k)}\right)$, which can be treated as the generalization of the Chudnovsky Conjecture and fits perfectly with (1):

$$
\alpha\left(I^{(3 r-k)}\right) \geq r \alpha(I)+2 r-k .
$$

Observe also that the containment $I^{(3 r-3)} \subset M^{2 r-3} I^{r}$ does not hold for $r=1$ (in fact we must define $M^{-1}$ in this case), so we focus on the case $I^{(3 r-k)} \subset M^{2 r-k} I^{r}$ for $k=0,1,2$ and $r \geq 1$. There are also examples when the containment $I^{(2 r)} \subset I^{r}$ does not hold (see Corollary 1.1.1 in Boc-Har 10a]).

We need to introduce the Waldschmidt constant of an ideal (cf. Har-Hun 11]).

Proposition 5. Let $I \subset K\left[\mathbb{P}^{N}\right]$ be a non-zero homogeneous ideal. Define the Waldschmidt constant

$$
\gamma(I)=\lim _{m \longrightarrow \infty} \frac{\alpha\left(I^{(m)}\right)}{m} .
$$

Then the following holds:

(1) $\gamma(I)$ exists and satisfies $\alpha\left(I^{(m)}\right) \geq m \gamma(I)$ for all $m \geq 1$;

(2) if $I \subset J$ are ideals of finite number of points, then $\gamma(I) \geq \gamma(J)$;

(3) if $I$ is an ideal of fat points, then $\gamma\left(I^{(r)}\right)=r \gamma(I)$.

Proof. See [Dum 11, Proposition 7] and [Boc-Har 10a, Lemma 2.3.1].

Define $\gamma(N, n)=\gamma(I)$, where $I$ is the ideal of $n$ generic points in $\mathbb{P}^{N}$. The conjectural value for $\gamma(N, n)$ is $\sqrt[N]{n}$ for $n$ big enough. Anyway, we always have the inequality $\gamma(N, n) \leq \sqrt[N]{n}$ (see [DHST 12]). Therefore, for $n$ big, we are interested in lower bounds for $\gamma(N, n)$. Since we deal with the case $N=3$ we will write $\gamma(n)=\gamma(3, n)$. As a by-product we will show the following:

$$
\gamma(n) \geq 0.7787 \sqrt[3]{n}+0.6142, \quad \text { for } n \geq 512 .
$$

\section{Bounds For the WALDschmit CONSTANt}

Proposition 6. Let $\bar{m}$ be a sequence of multiplicities. If

$$
I\left(m_{1}, m_{2}, m_{3}, m_{4}, \bar{m}\right)_{t} \neq 0,
$$

then

$$
I\left(m_{1}+k, m_{2}+k, m_{3}+k, m_{4}+k, \bar{m}\right)_{t+k} \neq 0
$$

for $k=2 t-m_{1}-m_{2}-m_{3}-m_{4}$.

Proof. The proof involves using standard birational transformation for $\mathbb{P}^{3}$ and some combinatorial arguments to deal with the case of negative multiplicities. The full proof can be found in Dum 11] (see Proposition 8 there).

The above operation on ideals and degrees will be called a "Cremona operation", or simply a "Cremona". Since we can permute multiplicities, a Cremona operation can be performed on any four of them. In such situations, we will indicate on which multiplicities (or on which points) it is performed. 
Corollary 7. With notation as above,

$\operatorname{dim}_{\mathbb{K}} I\left(m_{1}, m_{2}, m_{3}, m_{4}, \bar{m}\right)_{t}=\operatorname{dim}_{\mathbb{K}} I\left(m_{1}+k, m_{2}+k, m_{3}+k, m_{4}+k, \bar{m}\right)_{t+k}$.

Proof. Observe that $\operatorname{dim}_{\mathbb{K}} I\left(m_{1}, m_{2}, m_{3}, m_{4}, \bar{m}\right)_{t}$ is equal to the minimal number $v$ satisfying

$$
I\left(m_{1}, m_{2}, m_{3}, m_{4}, \bar{m}, 1^{\times v}\right)_{t}=0 .
$$

Proposition 8. Let $m_{1}, \ldots, m_{r}, t$ be integers. If

$$
4 t>m_{1}+\ldots+m_{8},
$$

then $I\left(m_{1}, \ldots, m_{r}\right)_{t}$ can be transformed by a sequence of Cremona operations into $I\left(m_{1}^{\prime}, \ldots, m_{r}^{\prime}\right)_{t^{\prime}}$ with arbitrarily large $t^{\prime}$. Moreover, these Cremona operations can be chosen to be applied only to $m_{1}, \ldots, m_{8}$.

Proof. Let $I(0)=I\left(m_{1}, \ldots, m_{r}\right)_{t}$, and define inductively $I(n)$ to be the space obtained from $I(n-1)$ by performing Cremona on multiplicities numbers $1,2,3$, 4 and again Cremona on multiplicities numbers 5, 6, 7, 8. Let $T(n)$ be the degree of $I(n)$, let $S_{1}(n)$ be the sum of the first four multiplicities in $I(n)$, and let $S_{2}(n)$ be the sum of the next four multiplicities. Denote also $s_{1}=m_{1}+m_{2}+m_{3}+m_{4}$, $s_{2}=m_{5}+m_{6}+m_{7}+m_{8}$. Obviously $T(0)=t, S_{1}(0)=s_{1}, S_{2}(0)=s_{2}$. Perform Cremona on $I(n-1)$ on the first four multiplicities. The new degree is equal to $3 T(n-1)-S_{1}(n-1)$, and the new sum of the first four multiplicities is equal to $8 T(n-1)-3 S_{1}(n-1)$. Performing another Cremona on the other four multiplicities gives recurrence relations for $T(n), S_{1}(n)$ and $S_{2}(n)$ :

$$
\begin{aligned}
T(n) & =9 T(n-1)-3 S_{1}(n-1)-S_{2}(n-1), \\
S_{1}(n) & =8 T(n-1)-3 S_{1}(n-1), \\
S_{2}(n) & =24 T(n-1)-8 S_{1}(n-1)-3 S_{2}(n-1) .
\end{aligned}
$$

We claim that

$$
\begin{aligned}
T(n) & =\left(8 n^{2}+1\right) t-\left(2 n^{2}+n\right) s_{1}-\left(2 n^{2}-n\right) s_{2}, \\
S_{1}(n) & =\left(16 n^{2}-8 n\right) t-\left(4 n^{2}-1\right) s_{1}-\left(4 n^{2}-4 n\right) s_{2}, \\
S_{2}(n) & =\left(16 n^{2}+8 n\right) t-\left(4 n^{2}+4 n\right) s_{1}-\left(4 n^{2}-1\right) s_{2} .
\end{aligned}
$$

Indeed, it is easy to observe that these polynomials evaluated at 0 give $t, s_{1}, s_{2}$ respectively and (using any computer algebra system) that they satisfy recurrence relations (21), (3) and (41). Since

$$
T(n)=\left(8 t-2 s_{1}-2 s_{2}\right) n^{2}+\text { lower terms }
$$

and $8 t-2 s_{1}-2 s_{2}>0$ by assumption we get that $T(n)$ can take arbitrarily large values for $n \gg 0$.

Proposition 9. Let $I\left(M_{1}(n), \ldots, M_{10}(n)\right)_{T(n)}$ be the space obtained from $I\left(0^{\times 10}\right)_{1}$ by $n$ iterations of the following operation: Cremona based on points numbers 1, 2, 3,4 , then $1,5,6,7$, then $1,8,9,10$. Then $\lim T(n)=\infty$ for $n \longrightarrow \infty$. 
Proof. For simplicity we change the notation and assume that

$$
I_{0}=I\left(0,0^{\times 3}, 0^{\times 3}, 0^{\times 3}\right){ }_{1},
$$

while $I(n)$ comes from $I(n-1)$ by six Cremona operations based on points: numbers $1,2,3,4$, then $1,5,6,7$, then 1, 8, 9, 10, then (again) 1, 2, 3, 4, then 1, 5, 6, 7, then $1,8,9,10$. We claim that

$$
I(n)=I\left(54 n^{2},\left(18 n^{2}-6 n\right)^{\times 3},\left(18 n^{2}\right)^{\times 3},\left(18 n^{2}+6 n\right)^{\times 3}\right)_{54 n^{2}+1} .
$$

The straightforward inductive proof of this fact is left to the reader.

Proposition 10. Let $m_{1}, \ldots, m_{r}, m_{1}^{\prime}, \ldots, m_{s}^{\prime}, t, k$ be integers. If

$$
I\left(m_{1}, \ldots, m_{r}\right)_{k}=0
$$

and

$$
I\left(m_{1}^{\prime}, \ldots, m_{s}^{\prime}, k+1\right)_{t}=0
$$

then

$$
I\left(m_{1}, \ldots, m_{r}, m_{1}^{\prime}, \ldots, m_{s}^{\prime}\right)_{t}=0 .
$$

Proof. [Dum 09, Theorem 10].

Proposition 11. Define $b(n)$ for the following values on $n$ :

\begin{tabular}{c|cccccccccccccccc}
$n$ & 1 & 2 & 3 & 4 & 5 & 6 & 7 & 8 & 12 & 14 & 16 & 17 & 21 & 24 & 30 & 35 \\
\hline$b(n)$ & 1 & 1 & 1 & $\frac{4}{3}$ & $\frac{5}{3}$ & $\frac{12}{7}$ & $\frac{28}{15}$ & 2 & $\frac{126}{57}$ & $\frac{7}{3}$ & $\frac{22}{9}$ & $\frac{5}{2}$ & $\frac{8}{3}$ & $\frac{107}{39}$ & 3 & $\frac{67}{21}$.
\end{tabular}

Then $\gamma(n) \geq b(n)$ whenever $b(n)$ is defined.

Proof. We will consider each case separately. We note here that for $n \leq 8$ we have $\gamma(n)=b(n)$. Moreover, for $n \leq 8$ this equality can be derived from a much more general procedure of computing $\alpha\left(I^{(m)}\right)$ for an ideal $I$ of at most 8 generic points in $\mathbb{P}^{3}$ and any $m \geq 1$ (see DV-La 07]).

- $n=1,2,3$ is clear.

- $n=4$. Let $I$ be the ideal of 4 generic points. If $\left(I^{(3 m)}\right)_{4 m-1} \neq 0$, then, by Proposition $6, I\left((-m-2)^{\times 4}\right)_{-2}=\mathbb{K}\left[\mathbb{P}^{3}\right]_{-2} \neq 0$, which is false. Hence $\alpha\left(I^{(3 m)}\right) \geq 4 m$ gives $\gamma(I) \geq \frac{4}{3}$ by Proposition 5 .

- $n=5$. Similarly, let $I$ be the ideal of 5 generic points. If $\left(I^{(3 m)}\right)_{5 m-1} \neq 0$, then, by Proposition $6, I\left(3 m,(-m-2)^{\times 4}\right)_{3 m-2} \neq 0$, which is false. Hence $\alpha\left(I^{(3 m)}\right) \geq 5 m$ gives $\gamma(I) \geq \frac{5}{3}$.

- $n=6$. Consider $I\left((7 m)^{\times 6}\right)_{12 m-1}$. We make the following Cremona transformations:

\begin{tabular}{c|cccccc|c}
$t$ & $m_{1}$ & $m_{2}$ & $m_{3}$ & $m_{4}$ & $m_{5}$ & $m_{6}$ & $k$ \\
\hline $12 m-1$ & $\underline{7 m}$ & $\underline{7 m}$ & $\underline{7 m}$ & $\underline{7 m}$ & $7 m$ & $7 m$ & $-4 m-2$ \\
$8 m-3$ & $3 m-2$ & $3 m-2$ & $\underline{3 m-2}$ & $\underline{3 m-2}$ & $\underline{7 m}$ & $\underline{7 m}$ & $-4 m-2$ \\
$4 m-5$ & $3 m-2$ & $\underline{3 m-2}$ & & & $\underline{3 m-2}$ & $\underline{3 m-2}$ & $-4 m-2$ \\
-7 & & & & & & &
\end{tabular}

to show that this space is zero.

- $n=7$. Consider $I\left((15 m)^{\times 7}\right)_{28 m-1}$. Again we make a Cremona transformation. To make the presentation better, we first show what happens with coefficients standing by $m$ 's, and then what happens with free coefficients. In other words, we make several Cremona on $I\left(15^{\times 7}\right)_{28}$ obtaining $I\left((-1)^{\times 7}\right)_{0}$, and then the same sequence of Cremona on $I\left(0^{\times 7}\right)_{1}$ obtaining 
$I\left((8)^{\times 4}, 4,8,8\right)_{15}$. By linearity, we obtain that $I\left((15 m)^{\times 7}\right)_{28 m-1}$ can be transformed into $I\left((-m-8)^{\times 6},-m-4\right)_{-15}$, which is zero.

\begin{tabular}{c|ccccccc|c}
$t$ & $m_{1}$ & $m_{2}$ & $m_{3}$ & $m_{4}$ & $m_{5}$ & $m_{6}$ & $m_{7}$ & $k$ \\
\hline 28 & $\underline{15}$ & $\underline{15}$ & $\underline{15}$ & $\underline{15}$ & 15 & 15 & 15 & -4 \\
24 & $\underline{11}$ & 11 & 11 & 11 & $\underline{15}$ & $\underline{15}$ & $\underline{15}$ & -8 \\
16 & 3 & $\underline{11}$ & $\underline{11}$ & $\underline{11}$ & $\underline{7}$ & $\frac{7}{3}$ & -8 \\
8 & $\underline{3}$ & $\underline{3}$ & 3 & -1 & $\underline{7}$ & $\underline{7}$ & -4 \\
4 & -1 & -1 & $\underline{3}$ & $\underline{3}$ & -1 & $\underline{3}$ & $\underline{3}$ & -4 \\
0 & -1 & -1 & -1 & -1 & -1 & -1 & -1 & \\
$t$ & $m_{1}$ & $m_{2}$ & $m_{3}$ & $m_{4}$ & $m_{5}$ & $m_{6}$ & $m_{7}$ & $k$ \\
\hline 1 & $\underline{0}$ & $\underline{0}$ & $\underline{0}$ & $\underline{0}$ & 0 & 0 & 0 & +2 \\
3 & $\underline{2}$ & 2 & 2 & 2 & $\underline{0}$ & $\underline{0}$ & $\underline{0}$ & +4 \\
7 & 6 & $\underline{2}$ & $\underline{2}$ & $\underline{2}$ & $\underline{4}$ & 4 & 4 & +4 \\
11 & $\underline{6}$ & $\underline{6}$ & 6 & 6 & 4 & $\underline{4}$ & $\underline{4}$ & +2 \\
13 & 8 & 8 & $\underline{6}$ & $\underline{6}$ & 4 & $\underline{6}$ & $\underline{6}$ & +2 \\
15 & 8 & 8 & 8 & 8 & 4 & 8 & 8 &
\end{tabular}

- $n=8$. Consider $I\left(m^{\times 8}\right)_{2 m-1}$. Observe that each Cremona on $I\left(1^{\times 8}\right)_{2}$ gives the same space. Hence we are interested only in the free part $I\left(0^{\times 8}\right)_{-1}$. By Proposition 8 the space $I\left(0^{\times 8}\right)_{1}$ can be transformed into some space with sufficiently large degree (greater than $m$ ); hence $I\left(m^{\times 8}\right)_{2 m-1}$ can be transformed into $I(\ldots)_{<0}$, which completes the proof of this case.

- $n=12$. Consider $I\left((57 m)^{\times 12}\right)_{126 m-1}$. Since $\gamma(5) \geq \frac{5}{3}$ we have, by Proposition 5, $\alpha\left(I\left((57 m)^{\times 5}\right)\right) \geq 95 m$; hence

$$
I\left((57 m)^{\times 5}\right)_{95 m-1}=0 .
$$

By Proposition 10 it suffices to show that $I\left(95 m,(57 m)^{\times 7}\right)_{126 m-1}=0$. Consider the following sequence of Cremona:

\begin{tabular}{c|cccccccc|c}
$t$ & $m_{1}$ & $m_{2}$ & $m_{3}$ & $m_{4}$ & $m_{5}$ & $m_{6}$ & $m_{7}$ & $m_{8}$ & $k$ \\
\hline 126 & $\underline{95}$ & $\underline{57}$ & $\underline{57}$ & $\underline{57}$ & 57 & 57 & 57 & 57 & -14 \\
112 & $\underline{\frac{}{43}}$ & 43 & 43 & $\underline{57}$ & $\underline{57}$ & $\underline{57}$ & 57 & -28 \\
84 & $\underline{53}$ & $\underline{43}$ & $\underline{43}$ & 43 & 29 & 29 & 29 & $\underline{57}$ & -28 \\
56 & 25 & 15 & 15 & $\underline{43}$ & $\underline{29}$ & $\underline{29}$ & $\underline{29}$ & $\frac{29}{11}$ & -18 \\
38 & $\underline{25}$ & $\underline{15}$ & 15 & $\underline{25}$ & 11 & 11 & $\underline{29}$ & -18 \\
20 & 7 & & $\underline{15}$ & $\underline{7}$ & $\underline{11}$ & $\underline{11}$ & $\underline{11}$ & 11 & -8 \\
12 & $\underline{7}$ & & $\underline{3}$ & $\underline{3}$ & $\underline{3}$ & $\underline{3}$ & $\underline{3}$ & -4 \\
4 & & & & & $\underline{3}$ &
\end{tabular}

Now perform the same sequence on the free part $I\left(0^{\times 8}\right)_{-1}$. Observe that $I\left(0^{\times 8}\right)_{1}$ is non-zero, so after any sequence of Cremona it will give a nonzero ideal, hence with positive degree (degree equal to 0 is excluded, since $\operatorname{dim}_{\mathbb{K}} I_{0} \leq 0$ but $\operatorname{dim}_{\mathbb{K}} I\left(0^{\times 8}\right)_{1}>0$; we use Corollary 7$)$. Therefore the free part will have negative degree, which completes the proof.

- $n=14$. Consider $I\left((3 m)^{\times 14}\right)_{7 m-1}$. It suffices to show that

$$
I\left(5 m,(3 m)^{\times 9}\right)_{7 m-1}=0 .
$$

Observe that Cremona based on multiplicities $5,3,3,3$ applied to $I\left(5,3^{\times 9}\right)_{7}$ gives exactly the same space, since $k=2 \cdot 7-5-3 \cdot 3=0$. Thus everything 
relies on the free part $I\left(0^{\times 10}\right)_{-1}$, which can have arbitrarily low degree according to Proposition 9 .

- $n=16$. Consider $I\left((9 m)^{\times 16}\right)_{22 m-1}$. It suffices to show that

$$
I\left(18 m,(9 m)^{\times 8}\right)_{22 m-1}=0 .
$$

Consider the following sequence of Cremona:

\begin{tabular}{c|ccccccccc|c}
$t$ & $m_{1}$ & $m_{2}$ & $m_{3}$ & $m_{4}$ & $m_{5}$ & $m_{6}$ & $m_{7}$ & $m_{8}$ & $m_{9}$ & $k$ \\
\hline 22 & $\underline{18}$ & $\underline{9}$ & $\underline{9}$ & $\underline{9}$ & 9 & 9 & 9 & 9 & 9 & -1 \\
21 & $\underline{17}$ & 8 & 8 & 8 & $\underline{9}$ & $\underline{9}$ & $\underline{9}$ & 9 & 9 & -2 \\
19 & $\underline{15}$ & $\underline{8}$ & 8 & 8 & 7 & 7 & 7 & $\underline{9}$ & $\underline{9}$ & -3 \\
16 & $\underline{12}$ & 5 & $\underline{8}$ & $\underline{8}$ & $\underline{7}$ & 7 & 7 & 6 & 6 & -3 \\
13 & $\underline{9}$ & 5 & 5 & 5 & 4 & $\underline{7}$ & $\underline{7}$ & $\underline{6}$ & 6 & -3 \\
10 & $\underline{6}$ & $\underline{5}$ & $\underline{5}$ & 5 & 4 & 4 & 4 & 3 & $\underline{6}$ & -2 \\
8 & $\underline{4}$ & 3 & 3 & $\underline{5}$ & $\underline{4}$ & $\underline{4}$ & 4 & 3 & 4 & -1 \\
7 & $\underline{3}$ & 3 & 3 & $\underline{4}$ & 3 & 3 & $\underline{4}$ & 3 & $\underline{4}$ & -1 \\
6 & 2 & 3 & 3 & 3 & 3 & 3 & 3 & 3 & 3 &
\end{tabular}

Observe that further Cremona based on points other than one does not change the obtained space, so from this point only the free part will change. Perform the same sequence on $I\left(0^{\times 9}\right)_{1}$.

\begin{tabular}{c|ccccccccc|c}
$t$ & $m_{1}$ & $m_{2}$ & $m_{3}$ & $m_{4}$ & $m_{5}$ & $m_{6}$ & $m_{7}$ & $m_{8}$ & $m_{9}$ & $k$ \\
\hline 1 & $\underline{0}$ & $\underline{0}$ & $\underline{0}$ & $\underline{0}$ & 0 & 0 & 0 & 0 & 0 & +2 \\
3 & $\underline{2}$ & 2 & 2 & 2 & $\underline{0}$ & $\underline{0}$ & $\underline{0}$ & 0 & 0 & +4 \\
7 & $\underline{6}$ & $\underline{2}$ & 2 & 2 & 4 & 4 & 4 & $\underline{0}$ & $\underline{0}$ & +6 \\
13 & $\underline{12}$ & 8 & $\underline{2}$ & $\underline{2}$ & $\underline{4}$ & 4 & 4 & 6 & 6 & +6 \\
19 & $\underline{18}$ & 8 & 8 & 8 & 10 & $\underline{4}$ & $\underline{4}$ & $\underline{6}$ & 6 & +6 \\
25 & $\underline{24}$ & $\underline{8}$ & $\underline{8}$ & 8 & 10 & 10 & 10 & 12 & $\underline{6}$ & +4 \\
29 & $\underline{28}$ & 12 & 12 & $\underline{8}$ & $\underline{10}$ & $\underline{10}$ & 10 & 12 & 10 & +2 \\
31 & $\underline{30}$ & 12 & 12 & $\underline{10}$ & 10 & 10 & $\underline{12}$ & 12 & $\underline{12}$ & -2 \\
29 & 28 & 12 & 12 & 8 & 10 & 10 & 10 & 12 & 10 &
\end{tabular}

We use Proposition 8 (for multiplicities $m_{2}, \ldots, m_{9}$ ) to complete the proof. - $n=17$. Consider $I\left((2 m)^{\times 17}\right)_{5 m-1}$. It suffices to show that

$$
I\left(4 m,(2 m)^{\times 9}\right)_{5 m-1}=0 .
$$

As in the case $n=14$, only the free part will change during Cremona. But this free part can achieve arbitrarily low degree according to Proposition 9 .

- $n=21$. Consider $I\left((3 m)^{\times 21}\right)_{8 m-1}$. As $\gamma(5) \geq \frac{5}{3}, I\left((3 m)^{\times 5}\right)_{5 m-1}=0$. We use Proposition 10 three times (on three different groups of five multiplicities each), which reduces the problem to showing $I\left(\left(5 m^{\times 3},(3 m)^{\times 6}\right)_{8 m-1}=\right.$ 0 . Observe that Cremona on $I\left(5^{\times 3}, 3^{\times 6}\right)_{8}$ gives $I\left(3^{\times 3}, 1,3^{\times 5}\right)_{6}$. Further Cremona on this space does nothing (whenever we omit a point with multiplicity one). Since the free part becomes $I\left((-2)^{\times 3},-2,0^{\times 6}\right)_{-3}$ and multiplicity -2 will not be used, we are done by Proposition 8

- $n=24$. Consider $I\left((39 m)^{\times 24}\right)_{107 m}$. By Proposition[10 used four times with $I\left((39 m)^{\times 5}\right)_{65 m-1}=0$, it suffices to show that $I\left((65 m)^{\times 4},(39 m)^{\times 4}\right)_{107 m}=$ 0 . It is easy to observe that by a sequence of four Cremona we obtain $I(\ldots)_{-5 m}$, which completes the proof. 
- $n=30$. Consider $I\left(m^{\times 30}\right)_{3 m-1}$. Since $I\left(m^{\times 8}\right)_{2 m-1}=0$, it suffices to show that

$$
I\left((2 m)^{\times 3}, m^{\times 6}\right)_{3 m-1}=0 .
$$

After one Cremona we obtain $I\left((m-2)^{\times 3}, m^{\times 5}\right)_{3 m-3}$ and argue similarly as in the case $n=8$.

- $n=35$. Consider $I\left((21 m)^{\times 35}\right)_{67 m-1}$. We use Proposition 10 four times: three times with $I\left((21 m)^{\times 8}\right)_{42 m-1}=0$, and once with $I\left((21 m)^{\times 5}\right)_{35 m-1}=$ 0 . Therefore it suffices to show that

$$
I\left((42 m)^{\times 3}, 35 m,(21 m)^{\times 6}\right)_{67 m-1}=0 .
$$

Consider the following sequence of Cremona:

\begin{tabular}{c|cccccccccc|c}
$t$ & $m_{1}$ & $m_{2}$ & $m_{3}$ & $m_{4}$ & $m_{5}$ & $m_{6}$ & $m_{7}$ & $m_{8}$ & $m_{9}$ & $m_{10}$ & $k$ \\
\hline 67 & $\underline{42}$ & $\underline{42}$ & $\underline{42}$ & $\underline{35}$ & 21 & 21 & 21 & 21 & 21 & 21 & -27 \\
40 & 15 & 15 & 15 & 8 & $\underline{21}$ & $\frac{21}{17}$ & $\underline{21}$ & $\frac{21}{17}$ & 21 & 21 & -4 \\
36 & 15 & 15 & 15 & 8 & $\underline{17}$ & $\underline{17}$ & $\frac{21}{13}$ & $\underline{21}$ & -4 \\
32 & 15 & 15 & 15 & 8 & 13 & 13 & $\underline{17}$ & $\underline{17}$ & $\underline{17}$ & -4 \\
28 & $\underline{15}$ & $\underline{15}$ & $\underline{15}$ & 8 & $\underline{13}$ & 13 & 13 & 13 & 13 & 13 & -2 \\
26 & 13 & 13 & 13 & 8 & 11 & 13 & 13 & 13 & 13 & 13 &
\end{tabular}

The same sequence on $I\left(0^{\times 10}\right)_{1}$ gives

\begin{tabular}{c|cccccccccc|c}
$t$ & $m_{1}$ & $m_{2}$ & $m_{3}$ & $m_{4}$ & $m_{5}$ & $m_{6}$ & $m_{7}$ & $m_{8}$ & $m_{9}$ & $m_{10}$ & $k$ \\
\hline 1 & $\underline{0}$ & $\underline{0}$ & $\underline{0}$ & $\underline{0}$ & 0 & 0 & 0 & 0 & 0 & 0 & +2 \\
3 & 2 & 2 & 2 & 2 & $\underline{0}$ & $\underline{0}$ & $\underline{0}$ & $\underline{0}$ & 0 & 0 & +6 \\
9 & 2 & 2 & 2 & 2 & $\underline{6}$ & $\underline{6}$ & 6 & 6 & $\underline{0}$ & $\underline{0}$ & +6 \\
15 & 2 & 2 & 2 & 2 & 12 & 12 & $\underline{6}$ & $\underline{6}$ & $\underline{6}$ & $\underline{6}$ & +6 \\
21 & $\underline{2}$ & $\underline{2}$ & $\underline{2}$ & 2 & $\underline{12}$ & 12 & 12 & 12 & 12 & 12 & +24 \\
45 & 26 & 26 & 26 & 2 & 36 & 12 & 12 & 12 & 12 & 12 &
\end{tabular}

Now we proceed as in the case $n=21$.

Proposition 12. We have the following inequalities:

- $\gamma(n) \geq \gamma(k)$ for $n \geq k$;

- $\gamma\left(a \cdot 8^{k}\right) \geq 2^{k} \cdot \gamma(a)$, for non-negative integers $a$ and $k$.

Proof. The first inequality is obvious. The second follows from the fact that $\gamma(8 b) \geq$ $2 \gamma(b)$ and induction. To prove the last inequality, observe that $I\left(m^{\times 8}\right)_{2 m-1}=0$ (since $\gamma(8) \geq 2$ ). Using Proposition 10 exactly $b$ times, we know that if $I\left((2 m)^{\times b}\right)_{t}$ $=0$, then $I\left(m^{\times 8 b}\right)_{t}=0$. This, together with Proposition 5 , leads to the following inequalities:

$$
\alpha\left(I\left(m^{\times 8 b}\right)\right) \geq \alpha\left(I\left((2 m)^{\times b}\right)\right) \geq 2 m \gamma(b) .
$$

Dividing both sides by $m$ and passing to the limit completes the proof. We should also observe that the equality $\gamma\left(8^{k}\right)=2^{k}$ follows directly from Evain 05 .

From the above proposition we easily derive the following lower bound for $\gamma(n)$ :

$$
\gamma(n) \geq \frac{1}{2} \sqrt[3]{n}
$$

Unfortunately, this bound is not sufficient to show the containment we need. But we can find better bounds. 
Proposition 13. Define

$$
\delta(n)=\frac{3 \sqrt[3]{6 n}+4.3}{7}
$$

If $n \geq 512$, then

$$
\gamma(n) \geq \delta(n) \geq 0.7787 \sqrt[3]{n}+0.6142 .
$$

Proof. First observe that $n \in\left[8^{k}, 8^{k+1}\right.$ ) for some $k \geq 3$. We will consider several cases, depending on the position of $n$ in the interval $\left[8^{k}, 8^{k+1}\right)$.

- $n \in\left[8^{k}, \frac{3}{2} \cdot 8^{k}\right]$. First, show that

$$
1 \geq \frac{3}{7} \sqrt[3]{9}+\frac{4.3}{7 \cdot 2^{k}}
$$

Indeed, this is true for $k=3$ and thus for any $k \geq 3$, since the right hand side of the inequality decreases when $k$ increases. By Proposition 12

$$
\gamma(n) \geq \gamma\left(8^{k}\right) \geq 2^{k} .
$$

Multiplying both sides of (5) by $2^{k}$ we get exactly

$$
2^{k} \geq \delta\left(\frac{3}{2} \cdot 8^{k}\right)
$$

Since $\delta(n)$ is increasing with respect to $n$, we complete the proof in this case. The rest of the proof will go exactly along the same lines.

- $n \in\left[\frac{3}{2} \cdot 8^{k}, 2 \cdot 8^{k}\right]$. Observe that

$$
\frac{63}{57} \geq \frac{3}{7} \sqrt[3]{12}+\frac{4.3}{7 \cdot 2^{k}}
$$

Hence

$$
\gamma(n) \geq \gamma\left(\frac{3}{2} \cdot 8^{k}\right)=\gamma\left(12 \cdot 8^{k-1}\right) \geq 2^{k-1} \gamma(12) .
$$

Now we use Proposition 11 to bound $\gamma(12)$ :

$$
2^{k-1} \gamma(12) \geq 2^{k} \frac{63}{57} \geq \delta\left(2 \cdot 8^{k}\right) \geq \delta(n) .
$$

We will use Proposition 11 in all further cases.

- $n \in\left[2 \cdot 8^{k}, 3 \cdot 8^{k}\right]$. Observe that

$$
\frac{11}{9} \geq \frac{3}{7} \sqrt[3]{18}+\frac{4.3}{7 \cdot 2^{k}}
$$

Hence

$\gamma(n) \geq \gamma\left(2 \cdot 8^{k}\right)=\gamma\left(16 \cdot 8^{k-1}\right) \geq 2^{k-1} \gamma(16) \geq 2^{k} \frac{11}{9} \geq \delta\left(3 \cdot 8^{k}\right) \geq \delta(n)$.

- $n \in\left[3 \cdot 8^{k}, 4 \cdot 8^{k}\right]$. Observe that

$$
\frac{4}{3} \geq \frac{3}{7} \sqrt[3]{24}+\frac{4.3}{7 \cdot 2^{k}}
$$

Hence

$$
\gamma(n) \geq \gamma\left(3 \cdot 8^{k}\right)=\gamma\left(24 \cdot 8^{k-1}\right) \geq 2^{k-1} \gamma(24) \geq 2^{k} \frac{4}{3} \geq \delta\left(4 \cdot 8^{k}\right) \geq \delta(n) .
$$


- $n \in\left[4 \cdot 8^{k}, 6 \cdot 8^{k}\right]$. Observe that

Hence

$$
\frac{3}{2} \geq \frac{3}{7} \sqrt[3]{36}+\frac{4.3}{7 \cdot 2^{k}}
$$

$\gamma(n) \geq \gamma\left(4 \cdot 8^{k}\right) \geq \gamma\left(30 \cdot 8^{k-1}\right) \geq 2^{k-1} \gamma(30) \geq 2^{k} \frac{3}{2} \geq \delta\left(6 \cdot 8^{k}\right) \geq \delta(n)$.

- $n \in\left[6 \cdot 8^{k}, 8 \cdot 8^{k}\right]$. Observe that

Hence

$$
\frac{5}{3} \geq \frac{3}{7} \sqrt[3]{48}+\frac{4.3}{7 \cdot 2^{k}}
$$

$$
\gamma(n) \geq \gamma\left(5 \cdot 8^{k}\right) \geq 2^{k} \gamma(5) \geq 2^{k} \frac{5}{3} \geq \delta\left(8 \cdot 8^{k}\right) \geq \delta(n) .
$$

\section{Containment Results}

Proposition 14. Let $n \geq 1$, let $I$ be an ideal of $n$ generic points, let

$$
\left(\begin{array}{l}
s \\
3
\end{array}\right)<n \leq\left(\begin{array}{c}
s+1 \\
3
\end{array}\right)
$$

and let $r \geq 1$. If either

$$
\gamma(n) \geq \frac{(s+1) r-2}{3 r-2}
$$

or

$$
\alpha\left(I^{(3 r-2)}\right) \geq(s+1) r-2,
$$

then $I^{(3 r-2)} \subset M^{2 r-2} I^{r}$ and $I^{(3 r-1)} \subset M^{2 r-1} I^{r}$ for all $r \geq 1$.

Proof. From Dum 11, proof of Proposition 12] it follows that the CastelnuovoMumford regularity $\operatorname{reg}(I)$ of $I$ is at most $s-1$ and $I$ is generated in degrees at most $s-1$.

By [Boc-Har 10a, Lemma 2.3.4], if $r \operatorname{reg}(I) \leq \alpha\left(I^{(m)}\right)$, then $I^{(m)} \subset I^{r}$. By (7)

$$
\alpha\left(I^{(3 r-1)}\right) \geq \alpha\left(I^{(3 r-2)}\right) \geq(s+1) r-2 \geq(s-1) r \geq \operatorname{reg}(I) r
$$

gives $I^{(3 r-2)} \subset I^{r}$ and $I^{(3 r-1)} \subset I^{r}$.

By an argument similar to that used in [Har-Hun 11, Lemma 4.6] if, for an ideal $I$ of points in $\mathbb{P}^{N}, I^{(m)} \subset I^{r}$ and

$$
\alpha\left(I^{(m)}\right) \geq r t+k
$$

for some $t$ such that $I$ is generated in degrees $t$ and less,

$$
I^{(m)} \subset M^{k} I^{r} .
$$

Taking $t=s-1$ and using (7) we have

$$
\alpha\left(I^{(3 r-2)}\right) \geq(s+1) r-2=(s-1) r+2(r-1) \geq r t+2 r-2,
$$

which proves $I^{(3 r-2)} \subset M^{2 r-2} I^{r}$. Now observe that (by taking the derivative)

$$
\alpha\left(I^{(3 r-1)}\right)>\alpha\left(I^{(3 r-2)}\right),
$$

and hence

$$
\alpha\left(I^{(3 r-1)}\right) \geq \alpha\left(I^{(3 r-2)}\right)+1 \geq r t+2 r-1,
$$


which gives $I^{(3 r-1)} \subset M^{2 r-1} I^{r}$.

Observe also that (7) follows from (6) by Proposition 5 .

Proposition 15. Let $I$ be an ideal of $n \geq 512$ generic points. Then $I^{(3 r-2)} \subset$ $M^{2 r-2} I^{r}$ for $r \geq 3$.

Proof. Take $s$ satisfying

$$
\left(\begin{array}{l}
s \\
3
\end{array}\right)<n \leq\left(\begin{array}{c}
s+1 \\
3
\end{array}\right) .
$$

Define, as before,

$$
\delta(n)=\frac{3 \sqrt[3]{6 n}+4.3}{7}
$$

By Proposition 13 we know that

$$
\gamma(I)=\gamma(n) \geq \delta(n) .
$$

By a straightforward computation we can show that for $r \geq 3$,

$$
\delta(n) \geq \frac{(\sqrt[3]{6 n}+2.1) r-2}{3 r-2} .
$$

From the inequality

$$
s(s-1)(s-2) \geq(s-1.1)^{3},
$$

which holds for $s \geq 5$, we obtain (since $6 n>s(s-1)(s-2)$ by (8) $)$

$$
\sqrt[3]{6 n}+1.1 \geq s
$$

and hence

$$
\frac{(\sqrt[3]{6 n}+2.1) r-2}{3 r-2} \geq \frac{(s+1) r-2}{3 r-2} .
$$

We conclude by Proposition 14 .

Proposition 16. Let $I$ be an ideal of $n$ generic points. If $5 \leq n \leq 511$, then $I^{(3 r-2)} \subset M^{2 r-2} I^{r}$ for $r \geq 3$.

Proof. Take $s$ satisfying

$$
\left(\begin{array}{l}
s \\
3
\end{array}\right)<n \leq\left(\begin{array}{c}
s+1 \\
3
\end{array}\right)
$$

(observe that $4 \leq s \leq 15$ ); we want to show that

$$
\gamma(n) \geq \frac{(s+1) r-2}{3 r-2}
$$

for all $r \geq 3$. Since $\gamma(n)$ is increasing (with respect to $n$ ) and the right hand side is decreasing (with respect to $r$ ), it is enough to show that

$$
\gamma\left(\left(\begin{array}{l}
s \\
3
\end{array}\right)+1\right) \geq \frac{3 s+1}{7}
$$


We will consider the following cases:

- $s=14$ and $s=15$. Since $\left(\begin{array}{c}14 \\ 3\end{array}\right)+1=365>320$, it suffices to show that $\gamma(320) \geq \frac{3 s+1}{7}$. But

$$
\gamma(320)=\gamma\left(5 \cdot 8^{2}\right) \geq 4 \gamma(5)
$$

by Proposition 12. Now we use Proposition 11 to obtain

$$
4 \gamma(5) \geq \frac{20}{3} \geq \frac{46}{7} \geq \frac{3 s+1}{7}
$$

All other cases will be treated similarly, with some changes for $s \leq 6$.

- $s=13$.

$$
\gamma(287) \geq \gamma(240) \geq 2 \gamma(30) \geq 6 \geq \frac{40}{7}=\frac{3 s+1}{7} .
$$

- $s=12$.

$$
\gamma(221) \geq \gamma(168) \geq 2 \gamma(21) \geq \frac{16}{3} \geq \frac{37}{7}=\frac{3 s+1}{7} .
$$

- $s=11$.

$$
\gamma(166) \geq \gamma(136) \geq 2 \gamma(17) \geq \frac{10}{2} \geq \frac{34}{7}=\frac{3 s+1}{7} .
$$

- $s=10$.

$$
\gamma(121) \geq \gamma(112) \geq 2 \gamma(14) \geq \frac{14}{3} \geq \frac{31}{7}=\frac{3 s+1}{7} .
$$

- $s=9$.

$$
\gamma(84) \geq \gamma(64) \geq 4 \gamma(1) \geq 4 \geq \frac{28}{7}=\frac{3 s+1}{7} .
$$

- $s=8$.

$$
\gamma(57) \geq \gamma(56) \geq 2 \gamma(7) \geq \frac{56}{15} \geq \frac{25}{7}=\frac{3 s+1}{7}
$$

- $s=7$.

$$
\gamma(36) \geq \gamma(35) \geq \frac{67}{21} \geq \frac{22}{7}=\frac{3 s+1}{7} .
$$

- $s=6$. Assume $n \geq 24$; then

$$
\gamma(n) \geq \gamma(24) \geq \frac{107}{39} \geq \frac{19}{7}=\frac{3 s+1}{7} .
$$


For $n \geq 21$ we have

$$
\gamma(n) \geq \gamma(21) \geq \frac{8}{3} \geq \frac{26}{10} \geq \frac{7 r-2}{3 r-2}
$$

for $r \geq 4$. For $r=3$, by Proposition 14 it is enough to show that (7) is satisfied, i.e.

$$
I\left(7^{\times 21}\right)_{18}=0 .
$$

By Proposition [10 it is enough to show that $I\left(14,14,7^{\times 5}\right)_{18}=0$. This can be done by Cremona operations.

- $s=5$. Assume $n \geq 14$; then

$$
\gamma(n) \geq \gamma(14) \geq \frac{7}{3} \geq \frac{16}{7}=\frac{3 s+1}{7} .
$$

For $n=12$ and $n=13$ we have

$$
\gamma(n) \geq \gamma(12) \geq \frac{107}{39} \geq \frac{22}{10} \geq \frac{6 r-2}{3 r-2}
$$

for $r \geq 4$. It remains to show the case $r=3$, i.e. that $\alpha\left(I\left(7^{\times 12}\right)\right) \geq 16$, but we will complete this together with the case $n=11$. For $n=11$ we want to show that

$$
\left.\alpha\left(I(3 r-2)^{\times 11}\right)\right) \geq 6 r-2 .
$$

By Proposition 10 it is enough to show that $I\left(4 r-2,(3 r-2)^{\times 7}\right)_{6 r-3}=0$. Consider the following Cremona operations:

\begin{tabular}{c|ccc|c}
$t$ & $m_{1}$ & $\left(m_{2}\right)^{\times 3}$ & $\left(m_{3}\right)^{\times 4}$ & $k$ \\
\hline $6 r-3$ & $\underline{4 r-2}$ & $\underline{3 r-2}$ & $3 r-2$ & $-r+2$ \\
$5 r-1$ & $3 r$ & $2 r$ & $\underline{3 r-2}$ & $-2 r+6$ \\
$3 r+5$ & $\underline{3 r}$ & $\underline{2 r}$ & $r+4$ & $-3 r+10$ \\
15 & 10 & $10-r$ & $\underline{r+4}$ & $-4 r+14$ \\
$29-4 r$ & 10 & $10-r$ & $18-3 r$ &
\end{tabular}

For $r \geq 5$ we have $29-4 r<10$, and then we are done. We are left with two cases to show:

$$
\begin{gathered}
\alpha\left(I\left(7^{\times 11}\right)\right) \geq 16, \\
\alpha\left(I\left(10^{\times 11}\right)\right) \geq 22 .
\end{gathered}
$$

The above cases will be completed in Lemma 19

- $s=4$. For $n \geq 7$ we have

$$
\gamma(n) \geq \gamma(7) \geq \frac{28}{15} \geq \frac{18}{10}=\frac{(s+1) 4-2}{3 \cdot 4-2} .
$$

Hence for $r \geq 4$ we are done; for $r=3$ we must show that

$$
\alpha\left(I\left(7^{\times n}\right)\right) \geq 13 .
$$

This is easy with Cremona operations. For $n=5$ and $n=6$ we will use DV-La 07]. Consider $I\left((3 r-2)^{\times 5}\right)_{5 r-3}$. By Cremona operation we get

$$
I\left(r^{\times 4}, 3 r-2\right)_{3 r-1} \text {. }
$$


Observe that there is no Cremona operation on multiplicities $r^{\times 4}, 3 r-2$ and degree $3 r-1$ which gives lower degree. Thus by [DV-La 07, Theorem 5.3] there is no element in $I\left(r^{\times 4}, 3 r-2\right)_{3 r-1}$ if and only if

$$
\left(\begin{array}{c}
3 r+2 \\
3
\end{array}\right)-4\left(\begin{array}{c}
r+2 \\
3
\end{array}\right)-\left(\begin{array}{c}
3 r \\
3
\end{array}\right)-1<0
$$

which is easy to compute. Hence $\alpha\left(I\left((3 r-2)^{\times n}\right)\right) \geq 5 r-2$, which completes the proof.

Proposition 17. Let $I$ be the ideal of $n \geq 65$ generic points. Then $I^{(3 r-2)} \subset$ $M^{2 r-2} I^{r}$ for $r=2$.

Proof. Take $s$ satisfying

$$
\left(\begin{array}{l}
s \\
3
\end{array}\right)<n \leq\left(\begin{array}{c}
s+1 \\
3
\end{array}\right)
$$

Observe that $s \geq 5$; hence

$$
\sqrt[3]{6 n}+1.1 \geq s
$$

By this inequality and Proposition 14 it is enough to show that

$$
\alpha\left(I\left(4^{\times n}\right)\right) \geq 2 \sqrt[3]{6 n}+2.2 .
$$

Take the least $t$ satisfying

$$
\left(\begin{array}{c}
t+3 \\
3
\end{array}\right) \geq 20 n
$$

By $\left[\mathrm{Ba}-\mathrm{Br} 09\right.$, Theorem 1] and [Dum 08, Theorem 3] there is no element in $I\left(4^{\times n}\right)_{t-1}$ if and only if

$$
\left(\begin{array}{c}
(t-1)+3 \\
3
\end{array}\right)-n\left(\begin{array}{l}
6 \\
3
\end{array}\right)-1<0
$$

Since

$$
(t+3)^{3} \geq(t+1)(t+2)(t+3) \geq 120 n
$$

we have that

$$
t \geq \sqrt[3]{120 n}-3 \geq 2 \sqrt[3]{6 n}+2.2
$$

for $n \geq 65$, which completes the proof.

Proposition 18. Let $I$ be an ideal of $n$ generic points. If $7 \leq n \leq 64$, then $I^{(3 r-2)} \subset M^{2 r-2} I^{r}$ for $r=2$.

Proof. Take $s$ satisfying

$$
\left(\begin{array}{l}
s \\
3
\end{array}\right)<n \leq\left(\begin{array}{c}
s+1 \\
3
\end{array}\right) .
$$

Then $4 \leq s \leq 8$. For each $s \geq 5$, we will show that

$$
I\left(4^{\times\left(\left(\begin{array}{l}
s \\
3
\end{array}\right)+1\right)}\right)_{2 s-1}=0 .
$$

Observe that by $\mathrm{Ba}-\mathrm{Br} 09$ and Dum 08, emptiness (i.e. containing no non-zero form) of $I\left(4^{\times n}\right)_{t}$ for $t \geq 9$ depends only on its "virtual dimension"

$$
v=\left(\begin{array}{c}
t+3 \\
3
\end{array}\right)-20 n \text {. }
$$


In each case $v$ becomes negative, which gives emptiness:

\begin{tabular}{cccc}
$s$ & $2 s-1$ & $\left(\begin{array}{l}s \\
3\end{array}\right)+1$ & $v$ \\
\hline 8 & 15 & 57 & -325 \\
7 & 13 & 36 & -161 \\
6 & 11 & 21 & -57 \\
5 & 9 & 11 & -1
\end{tabular}

Let $s=4, n \geq 7$. Use Cremona operations

\begin{tabular}{c|ccccccccc}
$t$ & $m_{1}$ & $m_{2}$ & $m_{3}$ & $m_{4}$ & $m_{5}$ & $m_{6}$ & $m_{7}$ & $m_{8} \ldots$ & $k$ \\
\hline 7 & $\underline{4}$ & $\underline{4}$ & $\underline{4}$ & $\underline{4}$ & 4 & 4 & 4 & $\ldots$ & -2 \\
5 & 2 & 2 & $\underline{2}$ & $\underline{2}$ & $\underline{4}$ & $\underline{4}$ & 4 & $\ldots$ & -2 \\
3 & 2 & 2 & 0 & 0 & 2 & 2 & 4 & $\ldots$ &
\end{tabular}

to show that $I\left(4^{\times n}\right)_{7}$ is the zero ideal.

Lemma 19. Let $I$ be the ideal of 4 or 5 generic points. Then

$$
I^{(4)} \subset M^{2} I^{2} .
$$

Moreover, if I is an ideal of 11 generic points, then

$$
\left(I^{(7)}\right)_{15}=\left(I^{(10)}\right)_{21}=0 .
$$

Proof. First of all, we may use the linear automorphism of $\mathbb{P}^{3}$ to assume that $p_{1}=(1: 0: 0: 0), p_{2}=(0: 1: 0: 0), p_{3}=(0: 0: 1: 0), p_{4}=(0: 0: 0: 1)$ (see Dum 11]). We take generic $p_{5}=(a: b: c: 1)$ and $p_{6}=(d: e: f: 1)$. Now we use the theory of Gröbner bases and Singular Sing to compute that $I^{(4)} \subset M^{2} I^{2}$ in the ring $\mathbb{K}(a, b, c, d, e, f)\left[\mathbb{P}^{3}\right]$ :

ring $R=(0, a, b, c, d, e, f),(x, y, z, w), d p ;$ option(redSB);

ideal $P 1=x, y, z$;

ideal $P 2=x, y, w$;

ideal $\mathrm{P} 3=\mathrm{x}, \mathrm{z}, \mathrm{w}$;

ideal $P 4=y, z, w$;

ideal $\mathrm{P} 5=\mathrm{x}-\mathrm{a} * \mathrm{w}, \mathrm{y}-\mathrm{b} * \mathrm{w}, \mathrm{z}-\mathrm{c} * \mathrm{w}$;

ideal $P 6=x-d * w, y-e * w, y-f * w$;

ideal $\mathrm{M}=\mathrm{x}, \mathrm{y}, \mathrm{z}, \mathrm{w}$;

ideal I5=intersect (P1, P2, P3, P4,P5);

ideal I6=intersect (I5,P6);

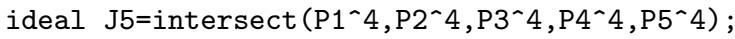

ideal J6=intersect (J5, $\left.\mathrm{P}^{\wedge}{ }^{\wedge}\right)$;

ideal $\mathrm{K} 5=$ quotient $\left(\mathrm{M}^{\wedge} 2 * \mathrm{I} 5^{\wedge} 2, \mathrm{~J} 5\right)$;

ideal $\mathrm{K} 6=$ quotient $\left(\mathrm{M}^{\wedge} 2 * \mathrm{I} 6^{\wedge} 2, \mathrm{~J} 6\right)$;

K5; K6 ;

$\mathrm{K} 5[1]=1$

$\mathrm{K} 6[1]=1$ 
All the computations performed by Singular will remain valid for generic values of parameters $a, b, c, d, e, f$ in $\mathbb{K}\left[\mathbb{P}^{3}\right]$; hence for generic $p_{5}$ and $p_{6}$ the answer would be the same, proving containment.

To show that $\left(I^{(7)}\right)_{15}=\left(I^{(10)}\right)_{21}=0$ we will find the element with the lowest degree in $I^{(7)}$ (resp. $I^{(10)}$ ), which can be done by computing the Gröbner basis of the ideal with respect to some degree ordering. By semicontinuity of $\alpha$, it is enough to compute the above for some chosen points. Moreover, non-existence of a non-zero element in the vector space $\left(I^{(7)}\right)_{15}$ is equivalent to maximality of the rank of a suitable interpolation matrix. But reduction modulo a prime number cannot make the rank bigger, so it is enough to perform computations over $\mathbb{Z} / p \mathbb{Z}$ for some prime $p$. Computations over $\mathbb{Z}$ have not finished within 24 hours.

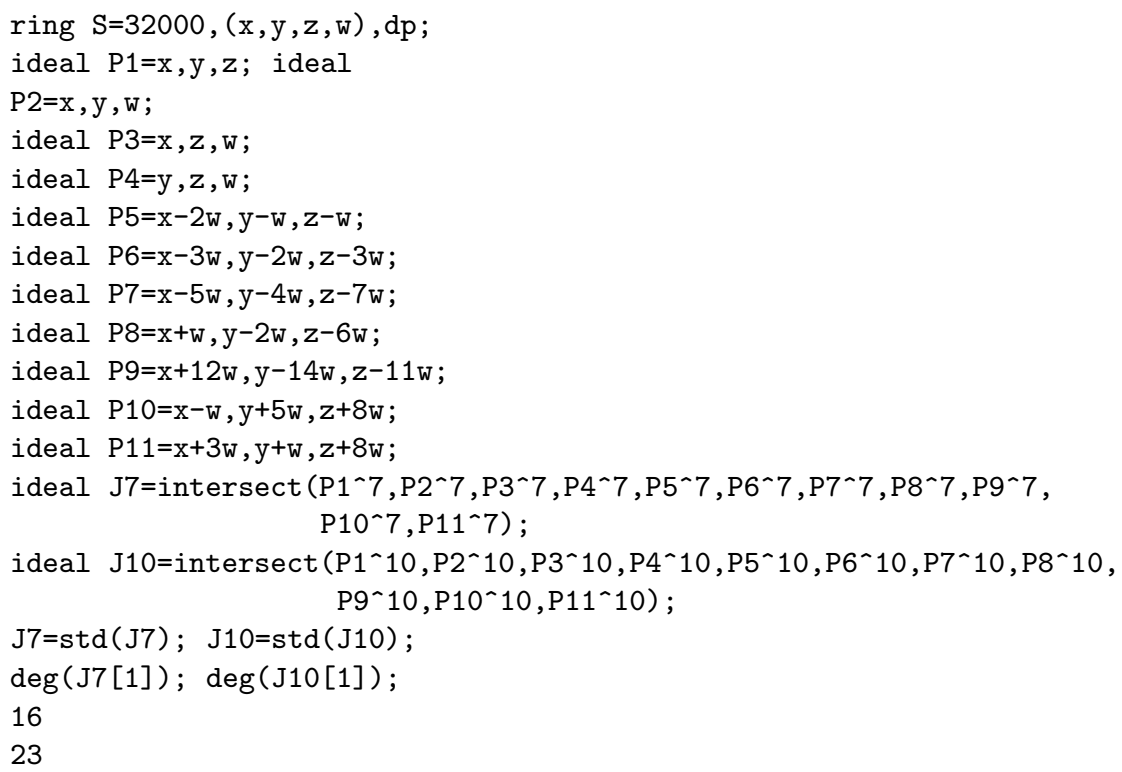

The case of $n \leq 4$ points will be solved using [Dum 11, Proposition 15]:

Proposition 20. Let $1 \leq n \leq N+1$, let $p_{1}=(1: 0: \ldots: 0), p_{2}=(0: 1: 0$ : $\ldots: 0), \ldots$ for $j=1, \ldots, n$ be fundamental points in $\mathbb{P}^{N}$, let $I=I\left(1^{\times n}\right)$, and let $J=\bigcap_{j=1}^{n} \mathfrak{m}_{p_{j}}$. Let $r \geq 1$. If $J^{(N r)} \subset M^{(N-1) r} J^{r}$, then $I^{(N r)} \subset M^{(N-1) r} I^{r}$.

For a sequence $\left(a_{0}, \ldots, a_{N}\right)$ of $N+1$ non-negative integers let

$$
x^{\left(a_{0}, \ldots, a_{N}\right)}=x_{0}^{a_{0}} \cdot \ldots \cdot x_{N}^{a_{N}} \in \mathbb{K}\left[\mathbb{P}^{3}\right] .
$$

Again, by [Dum 11, Proposition 16] we can describe generators of $I^{(m)}$ :

Proposition 21. Let $I$ be an ideal of $n \leq N+1$ fundamental points, and let $m \geq 1$. Then $\left(I^{(m)}\right)_{t}$ is generated by the following set of monomials:

$$
\mathcal{M}=\mathcal{M}(n, m)_{t}=\left\{x^{\left(a_{0}, \ldots, a_{N}\right)}: \sum_{j=0}^{N} a_{j}=t, a_{k} \leq t-m \text { for } k=0, \ldots, n-1\right\} .
$$

Proposition 22. Let $I$ be the ideal of at most four generic points in $\mathbb{P}^{3}$. Then $I^{(3 r-2)} \subset M^{2 r-2} I^{r}$ for all $r \geq 1$. 
Proof. The proof is very similar to the proof of Proposition 18 in Dum 11. Assume $r \geq 2$ and $n \geq 2$ ( $n$ denotes the number of points; for $n=1$ or $r=1$ the statement is clear). Choose $t \geq 0$; by Proposition 21 it is sufficient to show that every element from $\mathcal{M}$ belongs to $M^{2 r-2} I^{r}$. Let $x^{\left(a_{0}, \ldots, a_{3}\right)} \in \mathcal{M}$; of course $t \geq 3 r-2$. Our aim is to show that

$$
x^{\left(a_{0}, \ldots, a_{3}\right)}=y \cdot z,
$$

where $y$ is a product of $r$ monomials, each of them belonging to $I$, and $z$ is a monomial of degree at least $2 r-2$. Observe that, by Proposition 21, $x_{j} \in I$ for $j \geq n$, while for other indeterminates we have

$$
x_{j} x_{k} \in I \text { for } j<n, k<n .
$$

Hence $y$ should be equal to the product of $r$ factors, each of them being either a single indeterminate $x_{j}$ for $j \geq n$ or a product of two indeterminates $x_{j} x_{k}$ for $j, k<n$. Let

$$
s=\sum_{j=0}^{n-1} a_{j}, \quad p=\sum_{j=n}^{3} a_{j},
$$

and observe that $p+s=t$. If $p \geq r$, then $y$ can be taken to be a product of exactly $r$ indeterminates, so $\operatorname{deg} y=r$. Taking $z=x^{\left(a_{0}, \ldots, a_{3}\right)} / y$ we obtain

$$
\operatorname{deg} z=t-r \geq 3 r-2-r=2 r-2,
$$

hence $z \in M^{2 r-2}$. Now consider the case where $p<r$. Take $p$ single indeterminates of the form $x_{j}$ for $j \geq n$ and $2(r-p)$ indetermines of the form $x_{j}$ for $j<n$ in such a way that their product $y$ divides $x^{\left(a_{0}, \ldots, a_{3}\right)}$. It is possible since

$$
2(r-p)=2 r-2 p \leq t-r+2-2 p=(t-p)-r+2-p \leq s .
$$

Thus $y \in I^{r}$. Letting $z=x^{\left(a_{0}, \ldots, a_{3}\right)} / y$, we have

$$
\operatorname{deg}(z)=s-2(r-p) .
$$

Thus it suffices to show that

$$
s-2(r-p) \geq 2 r-2,
$$

which is equivalent to showing that

$$
t+p \geq 4 r-2 .
$$

For $t \geq 4 r-2$ this is obvious, so assume that $t<4 r-2$, ie. $t \leq 4 r-3$. Since $x^{\left(a_{0}, \ldots, a_{3}\right)} \in \mathcal{M}$ we have

$$
a_{j} \leq t-(3 r-2) \text { for } j=0, \ldots, n-1,
$$

hence

$$
s \leq n(t-(3 r-2)) .
$$

Therefore

$$
p \geq t-n(t-(3 r-2)),
$$

hence

$$
t+p \geq(2-n) t+3 n r-2 n .
$$


But

$$
(2-n) t \geq(2-n)(4 r-3)
$$

and thus

$$
t+p \geq(2-n)(4 r-3)+3 n r-2 n=(8-n) r+n-6 \geq 4 r-2,
$$

since $n=2,3$ or 4 .

\section{REFERENCES}

[Ba-Br 09] Edoardo Ballico and Maria Chiara Brambilla, Postulation of general quartuple fat point schemes in $\mathbf{P}^{3}$, J. Pure Appl. Algebra 213 (2009), no. 6, 1002-1012, DOI 10.1016/j.jpaa.2008.11.001. MR2498792 (2010b:14106)

[PSC 09] Thomas Bauer, Sandra Di Rocco, Brian Harbourne, Michał Kapustka, Andreas Knutsen, Wioletta Syzdek, and Tomasz Szemberg, A primer on Seshadri constants, Interactions of classical and numerical algebraic geometry, Contemp. Math., vol. 496, Amer. Math. Soc., Providence, RI, 2009, pp. 33-70, DOI 10.1090/conm/496/09718. MR2555949 (2010k:14010)

[Boc-Har 10a] Cristiano Bocci and Brian Harbourne, Comparing powers and symbolic powers of ideals, J. Algebraic Geom. 19 (2010), no. 3, 399-417, DOI 10.1090/S10563911-09-00530-X. MR2629595 (2011d:13021)

[Boc-Har 10b] Cristiano Bocci and Brian Harbourne, The resurgence of ideals of points and the containment problem, Proc. Amer. Math. Soc. 138 (2010), no. 4, 1175-1190, DOI 10.1090/S0002-9939-09-10108-9. MR2578512 (2011f:14010)

[Chud 81] G. V. Chudnovsky, Singular points on complex hypersurfaces and multidimensional Schwarz lemma, Seminar on Number Theory, Paris 1979-80, Progr. Math., vol. 12, Birkhäuser Boston, Mass., 1981, pp. 29-69. MR633888 (83m:32002)

[Sing] W. Decker, G.-M. Greuel, G. Pfister, and H. Schönemann, Singular 3-0-4 - A computer algebra system for polynomial computations. http://www.singular. uni-kl.de (2011).

[DV-La 07] Cindy De Volder and Antonio Laface, On linear systems of $\mathbb{P}^{3}$ through multiple points, J. Algebra 310 (2007), no. 1, 207-217, DOI 10.1016/j.jalgebra.2006.12.003. MR.2307790 (2008b:14009)

[Dum 08] Marcin Dumnicki, On hypersurfaces in $\mathbb{P}^{3}$ with fat points in general position, Univ. Iagel. Acta Math. 46 (2008), 15-19. MR2553357 (2011b:14017)

[Dum 09] Marcin Dumnicki, An algorithm to bound the regularity and nonemptiness of linear systems in $\mathbb{P}^{n}$, J. Symbolic Comput. 44 (2009), no. 10, 1448-1462, DOI 10.1016/j.jsc.2009.04.005. MR2543429 (2010i:14108)

[Dum 11] Marcin Dumnicki, Symbolic powers of ideals of generic points in $\mathbb{P}^{3}$, J. Pure Appl. Algebra 216 (2012), no. 6, 1410-1417, DOI 10.1016/j.jpaa.2011.12.010. MR 2890510

[DHST 12] M. Dumnicki, B. Harbourne, T. Szemberg, H. Tutaj-Gasi nska, Linear subspaces, symbolic powers and Nagata type conjectures, Adv. Math. 252 (2014), 471-491. MR3144238

[Ein-Laz-Smi 01] Lawrence Ein, Robert Lazarsfeld, and Karen E. Smith, Uniform bounds and symbolic powers on smooth varieties, Invent. Math. 144 (2001), no. 2, 241-252, DOI 10.1007/s002220100121. MR1826369(2002b:13001)

[Evain 05] Laurent Evain, On the postulation of $s^{d}$ fat points in $\mathbb{P}^{d}$, J. Algebra 285 (2005), no. 2, 516-530, DOI 10.1016/j.jalgebra.2004.09.034. MR2125451 (2005j:13019)

[Har-Hun 11] B. Harbourne, and C. Huneke, Are symbolic powers highly evolved?, J. Ramanujan Math. Soc. 28A (2013), 247-266. MR3115195

Institute of Mathematics, Jagiellonian University, Ul. Lojasiewicza 6, 30-348 KraKów, POLAND

E-mail address: Marcin.Dumnicki@im.uj.edu.pl 